\title{
Automated sleep apnea classification based on statistical and spectral analysis of electrocardiogram signals
}

\author{
Lavu Venkata Rajani Kumari ${ }^{1}$, Anumolu Lohitha ${ }^{2}$, Atluri Kavya ${ }^{3}$, Nallamothu Tarakeswar ${ }^{4}$ \\ ${ }^{1}$ Department of ECE, VNR Vignana Jyothi Institute of Engineering and Technology, Hyderabad, India \\ ${ }^{2}$ Catalog Specialist, Amazon, Hyderabad, India \\ ${ }^{3}$ Department of ECE, SRM Institute of Science and Technology, Chennai, India \\ ${ }^{4}$ Department of CSE, SRM Institute of Science and Technology, Chennai, India
}

\begin{tabular}{l} 
Article Info \\
\hline Article history: \\
Received Sep 17, 2021 \\
Revised Dec 20, 2021 \\
Accepted Jan 11, 2022 \\
\hline Keywords: \\
Discrete wavelets transform \\
random forest \\
Obstructive sleep apnea \\
electrocardiogram \\
Support vector machine
\end{tabular}

Corresponding Author:

Lavu Venkata Rajani Kumari

Department of ECE, VNR Vignana Jyothi Institute of Engineering and Technology

Hyderabad, Telangana-500090, India

Email: rajanikumar_lv@vnrvjiet.in

\begin{abstract}
Well-being sleep is a significant segment for maintaining mental comparably as genuine flourishing. More than six-hour recordings are required to distinguish sleep apnea, which are extremely long duration recordings. It's difficult for a human to deduce the problem from electrocardiogram (ECG) readings. As a result, automated PC-based assessment is expected to detect abnormalities as early as possible. An automated framework for the classification of obstructive sleep apnea (OSA) can moreover be distinguished from the ECG Signals. From the Massachusetts Institute of Technology-Beth Israel Hospital (MIT-BIH) polysomnographic informational collection. 18 subjects have been considered as data signals. The signal is segmented into 30 seconds and features are extracted by using the discrete wavelet transform (DWT). DWT of seven-level decomposition is applied on the segmented signal by using the wavelet 'sym3'. 12 features were extracted from each level and all of them are used to categorize the five types of sleep apnea. Random forest, k-nearest neighbor (KNN), and support vector machine $(\mathrm{SVM})$ are used for classification of apnea. The random forest (RF) classifier outperformed the others with an average of accuracy (Acc) of $98.86 \%$ according to the study's findings. The experimental results show the developed model outperforms the state of art algorithms in the literature.
\end{abstract}

This is an open access article under the CC BY-SA license.

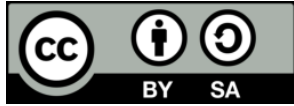

\section{INTRODUCTION}

Sleep assumes a significant job in human wellbeing. There is a development in time and cost of the unsafe results of poor sleep quality. Poor rest quality typically interfaces with physical and emotional wellness intricacies [1]. These apnea problems can have genuine and life-shortening results. Obstructive sleep apnea (OSA) is a condition in which the superior aeronautics closes temporarily during sleep, preventing air from inflowing the lungs [2]. When breathing does not yet come to a halt, the amount of air entering the lungs with each breath is reduced. The respiratory episode is recognised as hypopnea [3]. Different approaches for the detection of sleep apnea from electrocardiogram (ECG) signal have been proposed by researchers. The approaches start with data collecting from the Massachusetts Institute of Technology-Beth Israel Hospital (MITBIH) apnea database, data pre-processing, extraction of features from ECG signal and classification of features.

The variational mode decomposition is used to separate ECG into multiple modes, which are then utilised to extract various features and fed to the k-nearest neighbor classifier [4]. Different machine learning 
techniques are used for detection of sleep apnea like ANN [5]-[7], SVM [8]-[11], orthogonal wavelet filter bank [12]. Nishad et al. [13] uses ECG signal to identify apnea and non-apnea events. The ECG signal is decomposed into sub bands using wavelet transform, extracted features from each sub band and given the extracted features to different classifiers [13], [14]. A hidden Markov model is used for OSA detection that considers temporal dependence within segmented ECG signals [15]. The normal inverse Gaussian parameters for each sub-bands of ECG segments are computed, and adaptive boosting is used for detection of SA [16]. The beat-by-beat power spectral density of HRV and R peak area were evaluated using a bivariate autoregressive model. On a minute-by-minute basis for each recording, a k-nearest neighbor (KNN) was used to categorise apnea occurrences from normal ones [17]. ECG signals are commonly used to diagnose heart problems and sleep-related disorders. Various sources of noise within the signal's frequency band typically contaminate the recorded ECG signal, altering its properties and making it difficult to extract useable information from it [18]-[22]. The characteristics of the ECG signal are critical in identifying heart disorders [23]-[25] and sleep disorders. It is stated from the literature that most of the authors considered only two classes i.e., sleep apnea event and non-sleep apnea event. In our work, we have classified five types of slepp apnea (SA).

The objective of the project is to provide a computer-based solution for identifying sleep apnea using the machine learning algorithms. This research is targeted to achieve more accurate results compared to diagnosing with human interference. The general scope of this project is to identify five types of sleep apneas: Central apnea with arousal (CAA), obstructive apnea (OA), obstructive apnea with arousal (X), hypoapnea with arousal (HA), and normal (N) using available free MIT-BIH Polysomnographic. The features are extracted from the statistical analysis of discrete wavelet coefficients of collected ECG signal. The extracted features are given to the support vector machine (SVM), KNN and random forest for the classification of SA.

\section{METHOD}

\subsection{Dataset}

This data set was taken from the physionet by the Boston Beth Israel Hospital. The information utilized in this work taken from the MIT-BIH Polysomnographic database. This information base incorporates 18 subjects, where the individual record incorporates over 80 hours of ECG accounts and remarks on sleep stage and apnea labels. Every one of these signals was handled and examined at $250 \mathrm{~Hz}$. The ECG signal is available in the form of 30 seconds segments and each segment is labelled. There is an aggregate of 16 classes of sleep stage and apnea labels given in the data set, of which five sorts are considered for order in this examination as showed in Figure 1. We have extracted only these five-class labelled 30 seconds segments from each record and framed the data set. Figure 2 shows the normal ECG signal and the OSA issue in the ECG signal. The considered ECG signals are noise free. If the signal contains noise, then different noise removal algorithms will be implemented to remove the noise.

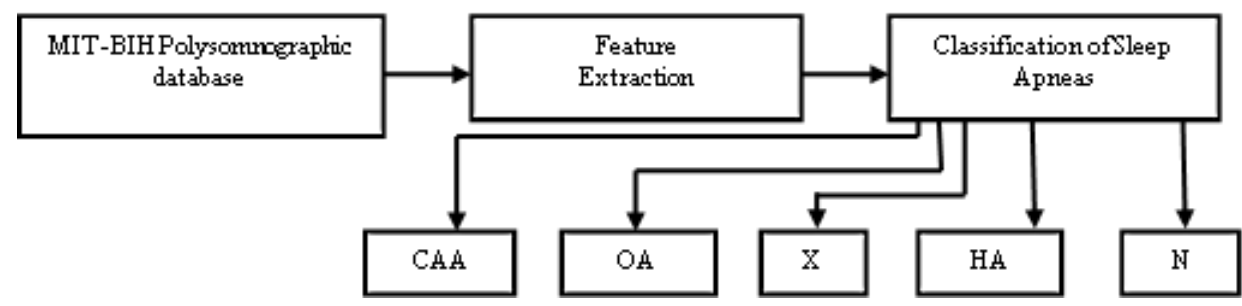

Figure 1. Sleep apnea classification block diagram

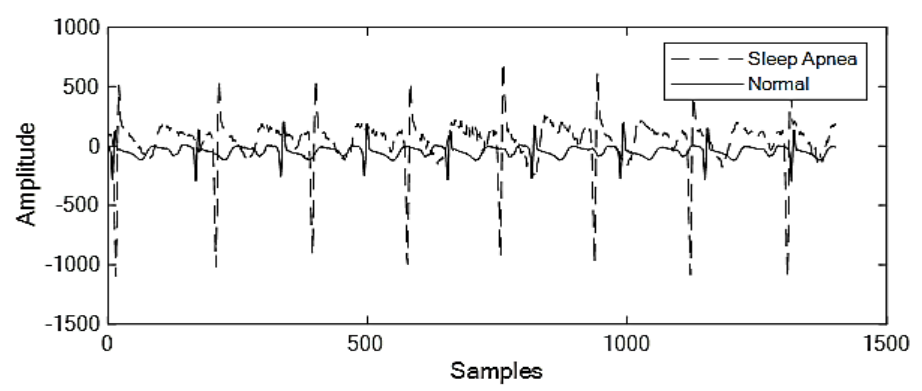

Figure 2. ECG signal 


\subsection{Feature extraction}

The following phase is to extract the features from ECG signals. Features address required characterization data for the signal. Later these separated features are utilized as the contributions to the classifiers. 18 records have been chosen from the MIT-BIH polysomnographic information base, with each signal portioned into 30 seconds. Wavelet transform works well with nonstationary signals like ECGs. It starts with a larger window to understand the major features, then moves on to a smaller window to recognise the smaller features. For tiny values of frequency, the wavelet transform has a high resolution in the frequency domain but a lower resolution in the time domain. However, it has a high resolution in the time domain at high frequencies but a low resolution in the frequency domain. This is the main reason wavelets are selected for feature extraction. To separate the features, a DWT of a 30-second epoch signal is used. The wavelet 'sym3' was used to complete a seven-level decomposition. The wavelet transform divides a signal into a set of approximate (Aj) and detailed (Dj) coefficients of level j=1, 2, ...7. Mean, correlation coefficient, standard deviation, skewness, variance, kurtosis, Shannon entropy, RMS, median, minimum wavelet coefficient, maximum wavelet coefficient and Harmonic mean were all considered in this work for the classification of apnea. For classification purposes, 12 features were collected from each level. The features are:

- Mean: It is defined as the average of the number of observations (N). The mean formula is given in (1).

$$
\mu=\frac{1}{N} \sum_{x=0}^{N} A x
$$

- Variance: It is determined by taking the normal squared deviations from the mean. Variance is given in (2):

$$
V=\frac{1}{N-1} \sum_{x=1}^{N}\left|A_{x}-\mu\right|^{2}
$$

where $\mu$ is the mean (2).

$$
\mu=\frac{1}{N} \sum_{x=0}^{N} A x
$$

- Standard deviation: It is the square root of the variance, and it is given in the formula as (3).

$$
\sigma=\sqrt{V}
$$

- Shannon entropy: Shannon entropy, otherwise called information entropy or the Shannon entropy record, is a proportion of the level of haphazardness in a bunch of information and it is given as (4).

$$
\begin{aligned}
H(i) & =\sum_{i}^{n} P(i) \cdot \log P(i) \\
& =\sum_{i}^{n} P(i) \cdot \log \left(\frac{1}{P(i)}\right)
\end{aligned}
$$

- Skewness: The skewness of a real-valued random variable's probability distribution around its mean is a measure of its asymmetry. It is given as:

$$
s=E[t-\mu]^{3} / \sigma^{3}
$$

here $\mu$ is the mean of $t, \sigma$ is the standard deviation of $t$.

- Kurtosis: It is an evaluation of how outlier-prone a distribution and it is given as (6):

$$
s=E[t-\mu]^{4} / \sigma^{4}
$$

where $\mu$ is the mean of $t, \sigma$ is the standard deviation of $t$.

- RMS: RMS is the root-mean-square value of a signal, and it is given as (7):

$$
\mathrm{X}_{\mathrm{RMS}}=\sqrt{\frac{1}{\mathrm{~N}} \sum_{\mathrm{n}=1}^{\mathrm{N}}\left|\mathrm{t}_{\mathrm{n}}\right|^{2}}
$$

- Median: It separates the higher half from the lower half of an information test, and it is given as:

$$
\operatorname{Med}(x)=\left\{\begin{array}{c}
x\left[\frac{n}{2}\right] \\
\frac{\left.\left(x \frac{n-1}{2}+x \frac{n+2}{2}\right]\right)}{2}
\end{array}\right.
$$

where $\mathrm{X}=$ set of values in the data set and $n=$ number of values. 
- Harmonic mean: The harmonic mean is frequently used to figure the normal of the proportions or rates. It is the most fitting measure for proportions and rates since it adjusts loads of every information point, and it is given in (9):

$$
\mathrm{m}=\frac{\mathrm{n}}{\sum_{\mathrm{i}=1 \frac{1}{\mathrm{x}}}^{\mathrm{n}}}
$$

where $\mathrm{n}$ is the number of values in $\mathrm{x}$.

- Correlation coefficient: It is characterized as the relationship coefficient of two irregular factors is a proportion of their straight reliance and it is given in (10):

$$
\rho(A, B)=\frac{1}{N-1} \sum_{i=1}^{n}\left(\frac{A-\mu}{\sigma}\right)\left(\frac{B-\mu}{\sigma}\right)
$$

where $\mu$ is the mean and $\sigma$ is the standard deviation.

\subsection{Classification}

In this work, machine learning algorithms like k-nearest neighbour, support vector machine and random forest are implemented to identify the five kinds of sleep apnea. Support vector machines are a type of coordinated learning algorithm that can be used for both classification and regression. The objective is to find a hyperplane in $\mathrm{n}$-dimensional space.

a. Support Vectors: Data focuses that are closest to the hyperplane are called support vectors. A segregating line will be portrayed with the help of these data focuses.

b. Hyperplane: a decision plane or space which is parted between lots of different classes.

c. Margin: It may be described as the gap between the two lines on the nearest data points of different classes. It might be resolved as the contrary partition from the line to the help vectors.

The key target of SVM is to separate the datasets into classes to find a maximum marginal hyperplane (MMH) and it will in general be done in the going with two phases: i) First, SVM will make hyperplanes iteratively that seclude the classes in the best way; and ii) Then, it will pick the hyperplane that disengages the classes successfully.

\subsubsection{K-Nearest Neighbors (KNN)}

KNN relies upon the k-number of closest neighbours. KNN is a non-parametric learning calculation and doesn't have any kind of assumptions regarding the info informational index and its appropriation. Whenever a data sample is to be categorized, the distances are figured, and afterward relying on the nearest points, the given data sample is predicted. The mathematical expression of distance measures is given as (11).

$$
\text { Euclidean Distance: } d(x, y)=\sqrt{\sum_{i=1}^{m}\left(a_{i}-b_{i}\right)^{2}}
$$

\subsubsection{Algorithm of KNN}

The whole process of KNN is explained as: i) Consider k number of nearest neighbors; ii) Euclidean Distance is calculated between $\mathrm{k}$ numbers of neighbors; iii) Calculate the data points among these $\mathrm{k}$ neighbors in each category; iv) The group of nearest neighbors is grouped; and v) The majority voting is used as the prediction of the given sample.

\subsubsection{Random forest (RF)}

With a bagging method, Random Forest creates classification trees based on randomly selected attributes of randomly selected samples. There is a direct correlation between the number of trees in the forest and the types of outcomes it can provide. Overfitting is a problem with many algorithms that can make the results worse, but the RF classifier will not overfit the model. Missing values can be handled with RF. Different trees are developed and during the checking, the votes from each tree are gathered and the target is by the predominance of votes.

\subsubsection{Algorithm of RF}

The whole process of RF is explained as: i) K-features are selected randomly from the training data set; ii) The decision tree is build based on the selected data points; iii) Select the number M to build the decision tree; iv) Steps i to ii are repeated; and v) Repeat steps i-iv for an n-number of times to generate an nnumber of trees, and the forest is complete. 


\subsection{Performance measures}

The following five performance parameters are utilized in this work: accuracy, sensitivity, specificity, precision, and F-score. The following performance parameters are computed from the confusion matrix.

- Accuracy: It's the number of successfully classified values divided by the total number of values.

$$
\text { Accuracy }=\frac{T P+T N}{T P+T N+F N+F P}
$$

- Precision: It is explained as the ratio of correct values to the total number of positive and false-positive values.

$$
\text { Precision }=\frac{T P}{T P+F P}
$$

- Sensitivity: Recall or Sensitivity is being explained as the ratio of correctly classified values to the sum of positive and wrongly detected values.

$$
\text { Sensitivity }=\frac{T P}{T P+F N}
$$

- Specificity: The fraction of no. of true negative predictions to total no. of negatives.

$$
\text { Specificity }=\frac{T N}{T N+F P}
$$

- F-Score: The harmonic mean of precision and recall is the F-Score.

$$
F-\text { Score }=2\left\{\frac{\text { precision } * \text { sensitivity }}{\text { precision }+ \text { sensitivity }}\right\}
$$

True positive (TP): Total number of correctly detected beats. False Positive (FP): Total number of missed beats. False negative (FN): Total number of wrongly detected. True Negative (TN): Total no of accurate negatively detected beats.

\section{RESULTS AND DISCUSSION}

Table 1 shows that the 18 signals result in a total of 9229 epochs. Total 96 features are extracted from each epoch (9229X96). There were 178 epochs of central apnea with arousal (CAA), 600 epochs of obstructive apnea (OA), 625 epochs of obstructive apnea with arousal (X), 740 epochs of hypoapnea with arousal (HA) arousal, and 7086 epochs of normal $(\mathrm{N})$. As a result, the complete feature set is separated into a training dataset $(80 \%)$ and a testing dataset $(20 \%)$ and distributed to the different classifiers. The Table 2 shows the correct classification and misclassification details.

Table 1. Total training and testing data set

\begin{tabular}{ccccc}
\hline S. No & OSA & Data Set & Training Data & Testing Data \\
\hline 1 & CAA & 178 & 143 & 35 \\
2 & OA & 600 & 480 & 120 \\
3 & X & 625 & 500 & 125 \\
4 & HA & 740 & 592 & 148 \\
5 & N & 7086 & 5668 & 1418 \\
& TOTAL & $\mathbf{9 2 2 9}$ & $\mathbf{7 3 8 6}$ & $\mathbf{1 8 4 6}$ \\
\hline
\end{tabular}

Table 2. Confusion matrix of three classifiers

\begin{tabular}{cccccccccccccccc}
\hline \multirow{2}{*}{ Sleep Apneas } & \multicolumn{4}{c}{ Confusion matrix of SVM } & \multicolumn{4}{c}{ Confusion matrix of KNN } & \multicolumn{4}{c}{ Confusion matrix of RF } \\
& $\mathrm{N}$ & HA & X & OA & CAA & N & HA & X & OA & CAA & N & HA & X & OA & CAA \\
\hline CAA & 0 & 1 & 0 & 0 & 34 & 0 & 1 & 0 & 0 & 34 & 0 & 0 & 0 & 0 & 35 \\
OA & 0 & 0 & 0 & 120 & 0 & 0 & 0 & 0 & 120 & 2 & 0 & 0 & 0 & 120 & 0 \\
X & 0 & 54 & 53 & 0 & 18 & 0 & 4 & 70 & 47 & 1 & 0 & 7 & 105 & 0 & 13 \\
HA & 0 & 146 & 0 & 0 & 2 & 0 & 146 & 0 & 0 & 2 & 0 & 147 & 0 & 0 & 1 \\
N & 1418 & 0 & 0 & 0 & 0 & 1418 & 0 & 0 & 0 & 0 & 1418 & 0 & 0 & 0 & 0 \\
\hline
\end{tabular}


The performance measures are computed using the confusion matrix of the classifiers and are listed in Table 3 for each sleep apnea. The average accuracy of SVM classifier is $95.93 \%$, KNN classifier is $96.85 \%$ and the Random Forest classifier is $98.86 \%$. Figure 3 shows the comparison of performance parameters of all classifiers.

Table 3. Performance parameters of classifiers

\begin{tabular}{ccccccccccccc}
\hline \multirow{2}{*}{ Sleep Apnea } & \multicolumn{4}{c}{ SVM Classifier } & \multicolumn{4}{c}{ KNN classifier } & \multicolumn{4}{c}{ RF classifier } \\
& Se $\%$ & Sp $\%$ & P $\%$ & Fs $\%$ & Se $\%$ & Sp $\%$ & P $\%$ & Fs $\%$ & Se $\%$ & Sp $\%$ & P\% & Fs $\%$ \\
\hline N & 100 & 100 & 100 & 100 & 100 & 100 & 100 & 100 & 100 & 100 & 100 & 100 \\
HA & 98.64 & 96.72 & 72.63 & 83.66 & 98.64 & 99.7 & 96.69 & 98.17 & 99.32 & 99.58 & 95.45 & 97.47 \\
X & 42.5 & 100 & 100 & 59.64 & 57.37 & 100 & 100 & 100 & 84 & 100 & 100 & 100 \\
OA & 100 & 100 & 100 & 100 & 98.36 & 97.26 & 81.63 & 88.76 & 100 & 100 & 100 & 100 \\
CAA & 97.14 & 99.94 & 62.96 & 76.4 & 97.14 & 99.72 & 87.17 & 93.02 & 100 & 99.22 & 64.15 & 77.92 \\
Avg & 87.65 & $\mathbf{9 9 . 3 3}$ & $\mathbf{8 7 . 1 2}$ & $\mathbf{8 3 . 9 4}$ & $\mathbf{9 0 . 3}$ & $\mathbf{9 9 . 3 4}$ & $\mathbf{9 3 . 1}$ & $\mathbf{9 5 . 9 9}$ & $\mathbf{9 6 . 6 6}$ & $\mathbf{9 9 . 7 6}$ & $\mathbf{9 1 . 9 2}$ & $\mathbf{9 5 . 0 8}$ \\
\hline
\end{tabular}

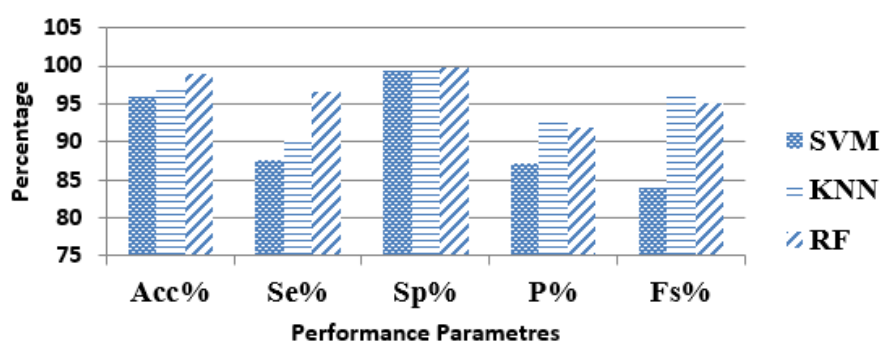

Figure 3. Performance comparison of classifiers

\subsection{Comparison with the literature}

Throughout the most recent couple of years, different analysts have proposed new strategies, methods that have been created for the recognizable proof of obstructive sleep apnea disorder. For the most part, the data sets considered are from Physio net and from different emergency clinics, which is compared with the proposed method as shown in Table 4. The performance of the Random Forest classifier with the chosen features provides better performance than the existing algorithms

Table 4. Comparison with the existing literature

\begin{tabular}{|c|c|c|c|c|c|c|}
\hline \multirow{2}{*}{ Authors } & \multirow{2}{*}{ Data input } & \multirow{2}{*}{ Features } & \multirow{2}{*}{ Classifiers } & \multicolumn{3}{|c|}{ Performance results } \\
\hline & & & & Acc $\%$ & Se $\%$ & Sp \% \\
\hline Ahsan et al. [27] & $\begin{array}{l}\text { Institute of breathing } \\
\text { and sleep Austin } \\
\text { Hospital }\end{array}$ & $\begin{array}{l}\text { Events of } \\
\text { Hypopnea and } \\
\text { DWT }\end{array}$ & $\begin{array}{l}\text { Two-staged } \\
\text { feed-forward } \\
\text { NN }\end{array}$ & 95 & 92 & 99 \\
\hline Majdi et al. [23] & Apnea ECG-Physionet & $\begin{array}{l}\text { Time and spectral } \\
\text { domain }\end{array}$ & SVM & 96 & - & - \\
\hline Lin et al. [24] & $\begin{array}{l}\text { MIT-BIH } \\
\text { database Physionet }\end{array}$ & Wavelet transform & ANN & - & 70 & 45 \\
\hline Carolina et al. [25] & $\begin{array}{l}\text { Apnea ECG-Physionet } \\
\text { and KU Leuven sleep } \\
\text { lab }\end{array}$ & Wavelet and HRV & Threshold & 85 & 85 & 85 \\
\hline Serein et al. [9] & MIT database & $\begin{array}{l}\text { Wavelet packet } \\
\text { decomposition of } \\
\text { HRV }\end{array}$ & Linear SVM & 93.3 & 90 & 100 \\
\hline Sunil et al. [10] & Apnea ECG-Physionet & $\begin{array}{l}\text { Gabor filter } \\
\text { responses }\end{array}$ & $\begin{array}{l}\text { Least square } \\
\text { SVM }\end{array}$ & 93.3 & - & - \\
\hline Proposed work & $\begin{array}{l}\text { MIT-BIH } \\
\text { Polysomnographic }\end{array}$ & Wavelet features & $\begin{array}{l}\text { Random } \\
\text { Forest }\end{array}$ & 98.86 & 96.66 & 99.76 \\
\hline
\end{tabular}

\section{CONCLUSION}

Automated sleep apnea classification models have been developed to classify five types of sleep apnea. ECG signals are collected from MIT-BIH Polysomnographic database, and the feature extraction is based on the DWT. Totally 12 features are extracted from each level and given as inputs to different classifiers. By comparing with various classifiers, random forest classifiers have shown better $98.86 \%$ 
accuracy, $96.66 \%$ sensitivity, $99.76 \%$ specificity, $91.92 \%$ of precision, and an overall F-score of $95.08 \%$, in detecting and classifying the sleep apnea from ECG signals.

\section{REFERENCES}

[1] A. Sheta, "Diagnosis of Obstructive Sleep Apnea from ECG Signals Using Machine Learning and Deep Learning Classifiers," Applied Science, vol. 11, no. 14, 2021, doi: 10.3390/app11146622.

[2] B. Medapati and L. V. Rajani Kumari, "KNN Based Sleep Apnea Detection Using ECG Signals," 2021 2nd International Conference for Emerging Technology (INCET), 2021, pp. 1-5, doi: 10.1109/INCET51464.2021.9456404.

[3] R. Rohan and L. V. R. Kumari, "Classification of Sleep Apneas using Decision Tree Classifier," 2021 th International Conference on Intelligent Computing and Control Systems (ICICCS), 2021, pp. 1310-1316, doi: 10.1109/ICICCS51141.2021.9432197.

[4] H. Sharma, K. K. Sharma, "Sleep apnea detection from ECG using variational mode decomposition," Biomed Phys Eng Express, vol. 6, no. 1, 2020, doi: 10.1088/2057-1976/ab68e9.

[5] J. Bali, A. Nandi, and P. S. Hiremath, "Efficient ANN Algorithms for Sleep Apnea Detection Using Transform Methods," Advancement of Machine Intelligence in Interactive Medical Image Analysis, pp. 99-152, 2019.

[6] S. Surani, A. Sheta, H. Turabieh, J. Park, S. Mathur, and A. Katangur, “A. Diagnosis of sleep apnea using artificial neural network and binary particle swarm optimization for feature selection," Chest Journal, 2019, vol. 156, no. 4, doi: 10.1016/j.chest.2019.08.215.

[7] T. Wang, C. Lu, and G. Shen, "Detection of Sleep Apnea from Single-Lead ECG Signal Using a Time Window Artificial Neural Network," BioMed Research International, vol. 2019, p. 9, 2019, doi: 10.1155/2019/9768072.

[8] H. Azimi, P. Xi, M. Bouchard, R. Goubran and F. Knoefel, "Machine Learning-Based Automatic Detection of Central Sleep Apnea Events from a Pressure Sensitive Mat," in IEEE Access, vol. 8, pp. 173428-173439, 2020, doi: 10.1109/ACCESS.2020.3025808.

[9] S. Al-Ratrout and A. Hossen, "Support vector machine of wavelet packet spectral features for identification of obstructive sleep apnea," 2018 5th International Conference on Electrical and Electronic Engineering (ICEEE), 2018, pp. 380-383, doi: 10.1109/ICEEE2.2018.8391366.

[10] T. S. Kumar and V. Kanhangad, "Gabor Filter-Based One-Dimensional Local Phase Descriptors for Obstructive Sleep Apnea Detection Using Single-Lead ECG," in IEEE Sensors Letters, vol. 2, no. 1, pp. 1-4, March 2018, Art no. 7000204, doi: 10.1109/LSENS.2018.2807584.

[11] F. Mendonça, S. S. Mostafa, A. G. Ravelo-García, F. Morgado-Dias and T. Penzel, "A Review of Obstructive Sleep Apnea Detection Approaches," in IEEE Journal of Biomedical and Health Informatics, vol. 23, no. 2, pp. 825-837, March 2019, doi: 10.1109/JBHI.2018.2823265.

[12] M. Sharma, M. Raval, U. R. Acharya, "A new approach to identify obstructive sleep apnea using an optimal orthogonal wavelet filter bank with ECG signals," Informatics Med. Unlocked, vol. 16, p. 100170, 2019, doi: 10.1016/j.imu.2019.100170.

[13] A. Nishad, R. B. Pachori, U. R. Acharya, "Application of TQWT based filter-bank for sleep apnea screening using ECG signals," Journal of Ambient Intelligence and Humanized Computing, 2018, doi: 10.1007/s12652-018-0867-3.

[14] R. Rachim, V. Pradana, L. Gang, and C. W.-Young, "Sleep apnea classification using ECG-signal wavelet-PCA features," Biomedical materials and engineering, 2014, doi: 10.3233/BME-141106.

[15] C. Song, K. Liu, X. Zhang, L. Chen and X. Xian, "An Obstructive Sleep Apnea Detection Approach Using a Discriminative Hidden Markov Model from ECG Signals," in IEEE Transactions on Biomedical Engineering, vol. 63, no. 7, pp. 1532-1542, July 2016, doi: 10.1109/TBME.2015.2498199.

[16] A. R. Hassan, "Computer-aided obstructive sleep apnea detection using normal inverse Gaussian parameters and adaptive boosting," Biomedical Signal Processing and Control, vol. 29, pp. 22-30, 2016, doi: 10.1016/j.bspc.2016.05.009.

[17] M. O. Mendez et al., "Detection of Sleep Apnea from surface ECG based on features extracted by an Autoregressive Model," 2007 29th Annual International Conference of the IEEE Engineering in Medicine and Biology Society, 2007, pp. 6105-6108, doi: 10.1109/IEMBS.2007.4353742.

[18] L. V. R. Kumari, Y. P. Sai, N. Balaji, and K. Viswada, "FPGA Based Arrhythmia Detection," Procedia Computer Science, vol. 57, pp. 970-979, 2015, doi: 10.1016/j.procs.2015.07.495.

[19] L. V. R. Kumari, Y. P. Sai, and N. Balaji, "R-Peak Identification in ECG Signals using Pattern-Adapted Wavelet Technique," IETE Journal of Research, doi: 10.1080/03772063.2021.1893229.

[20] L. V. R. Kumari, A. j. Sabavat and Y. P. Sai, "Performance Evaluation of Adaptive Filtering Algorithms for Denoising the ECG Signal," 2021 Second International Conference on Electronics and Sustainable Communication Systems (ICESC), 2021, pp. 1-5, doi: 10.1109/ICESC51422.2021.9532852

[21] H. Yoon, S. H. Hwang, J. -W. Choi, Y. J. Lee, D. -U. Jeong and K. S. Park, "Slow-Wave Sleep Estimation for Healthy Subjects and OSA Patients Using R-R Intervals," in IEEE Journal of Biomedical and Health Informatics, vol. 22, no. 1, pp. 119-128, Jan. 2018, doi: 10.1109/JBHI.2017.2712861.

[22] A. H. Khandoker, J. Gubbi and M. Palaniswami, "Automated Scoring of Obstructive Sleep Apnea and Hypopnea Events Using Short-Term Electrocardiogram Recordings," in IEEE Transactions on Information Technology in Biomedicine, vol. 13, no. 6, pp. 1057-1067, Nov. 2009, doi: 10.1109/TITB.2009.2031639.

[23] M. Bsoul, H. Minn and L. Tamil, "Apnea MedAssist: Real-time Sleep Apnea Monitor Using Single-Lead ECG," in IEEE Transactions on Information Technology in Biomedicine, vol. 15, no. 3, pp. 416-427, May 2011, doi: 10.1109/TITB.2010.2087386.

[24] R. Lin et al., "A new approach for identifying sleep apnea syndrome using wavelet transform and neural networks," Biomedical Engineering: Applications, Basics, and Communications, vol. 18. no. 3. pp. 138-143, 2006, doi: 10.4015/S1016237206000233.

[25] C. Varon, A. Caicedo, D. Testelmans, B. Buyse and S. Van Huffel, "A Novel Algorithm for the Automatic Detection of Sleep Apnea from Single-Lead ECG," in IEEE Transactions on Biomedical Engineering, vol. 62, no. 9, pp. 2269-2278, Sept. 2015, doi: 10.1109/TBME.2015.2422378. 


\section{BIOGRAPHIES OF AUTHORS}
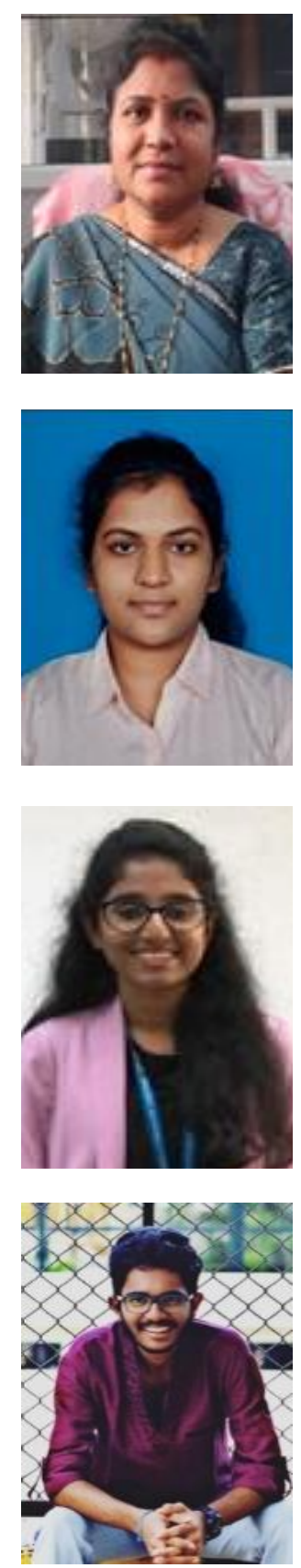

Lavu Venkata Rajani Kumari (iD) S SC P received B. Tech Degree in Electronics \& Communications Engineering, M. Tech degree in Embedded Systems and Ph.D. degree in Biomedical signal processing from the Jawaharlal Nehru Technological University of Hyderabad, India. Presently she is working as Assistant professor in the Department of ECE, VNRVJIET. She has presented 36 research papers in International Conferences/Journals. Her areas of research interest are Bio-Medical, Signal Processing and Embedded systems. She has received grants from AICTE to carry out research activities in the department. She is a member of IEEE, ISTE and IETE. She can be contacted at email: rajanikumari_lv@vnrvjiet.in.

Anumolu Lohitha (iD 8I SC P received B. Tech Degree in Electronics and Instrumentation Engineering in VNR Vignana Jyothi Institute of Engineering, Hyderabad, India. She has presented 02 research papers in International Conferences/Journals. Her areas of research interest are Bio-Medical, Signal Processing and Embedded systems. She can be contacted at email: lohithaanumolu@gmail.com.

Atluri Kavya (iD SC P is a student of SRM Institute of science and technology. She is currently pursuing her final year of bachelor's degree in the field of Elect. and communication engineering with specialization in biomedical engineering. Her research areas are biosensors, biomedical instrumentation and biomaterials. She can be contacted at email: atluri.kavya@gmail.com.

Nallamothu Tarakeswar (iD SA SC P is a student of SRM Institute of science and technology. $\mathrm{He}$ is currently pursuing his final year of bachelor's degree in the field of Computer Science engineering with specialization in Internet of Things. His research areas are Machine Learning and Deep Learning. He can be contacted at email: tarak0028@gmail.com. 